Case Report

\title{
Technical Feasibility of Acoustic Coordinated Reset Therapy for Tinnitus Delivered via Hearing Aids: A Case Study
}

\author{
Christian Hauptmann, ${ }^{1,2,3}$ Mark Williams, ${ }^{2,4}$ Federica Vinciati, ${ }^{2}$ and Markus Haller ${ }^{2,3}$ \\ ${ }^{1}$ Institute of Neuroscience and Medicine-Neuromodulation (INM-7), Jülich Research Center, Jülich, Germany \\ ${ }^{2}$ DESYNCRA Technologies Ltd., London, UK \\ ${ }^{3}$ DESYNCRA Operating GmbH, Bad Neuenahr-Ahrweiler, Germany \\ ${ }^{4}$ The Tinnitus Clinic Ltd., London, UK \\ Correspondence should be addressed to Christian Hauptmann; christian.hauptmann@desyncra.com
}

Received 30 November 2016; Revised 15 March 2017; Accepted 26 March 2017; Published 30 March 2017

Academic Editor: Nicolas Perez-Fernandez

Copyright (C) 2017 Christian Hauptmann et al. This is an open access article distributed under the Creative Commons Attribution License, which permits unrestricted use, distribution, and reproduction in any medium, provided the original work is properly cited.

\begin{abstract}
Primary tinnitus has a severe negative influence on the quality of life of a substantial portion of the general population. When acoustic coordinated reset (CR) neuromodulation stimuli are delivered for several hours per day over several weeks a clinically significant symptom reduction in patients with primary tonal tinnitus has been reported by several clinical sites. Here, we reported the first case where CR neuromodulation was delivered through a hearing aid. A 52-year-old man with chronic primary tonal tinnitus was previously considered untreatable with sound therapy. He initially received the classic CR treatment protocol with signals delivered with the separate proprietary device with his hearing aids removed during treatment. He was subsequently treated with the therapy being deployed through a set of contemporary hearing aids. After 5 months of classic CR treatment with the separate custom device, the THI and $\mathrm{VAS}_{\mathrm{L} / \mathrm{A}}$ scores worsened by $57 \%$ and $13 \% / 14 \%$, respectively. Using the hearing aid without CR treatment for 5 months no change in tinnitus symptoms was observed. However, after three months of CR treatment delivered through the hearing aids, the THI and $\mathrm{VAS}_{\mathrm{L} / \mathrm{A}}$ scores were reduced by $70 \%$ and $32 \% / 32 \%$, respectively.
\end{abstract}

\section{Introduction}

Primary (subjective) tinnitus is an idiopathic symptom that may or may not be associated with sensorineural hearing loss [1]. The prevalence of chronic primary tinnitus is $10 \%$ to $15 \%$ of the general population [2] with a range of severity. Approximately $2 \%$ of the general population experience a severely impaired quality of life due to this condition and seek professional help $[1,3]$. Secondary (objective) tinnitus refers to tinnitus associated with an identifiable organic condition other than sensorineural hearing loss [1]. Current tinnitus definitions and interventions are specified in detail in a widely accepted consensus guideline [4]. Accordingly, the only interventions with evidence-based outcomes are cognitive behavioral therapy [5], hearing aids [6], and sound therapy that can include sound maskers [7].

Sensorineural hearing loss, especially that associated with noise exposure and aging, can result in a reduction in the number of functioning sensory hair cells in the cochlea. Although sensory hair cell loss can lead to a sensorineural hearing loss, it is highly unlikely that the hair cell loss itself is the source of the tinnitus. It is much more likely that the deafferentation process can trigger abnormal neuronal synchrony in the central nervous system and, if persistent, can lead to an abnormal upregulation of synaptic connectivity to other brain regions resulting in chronic primary tinnitus [8-10]. Chronic primary tinnitus is associated with altered spectral power of electroencephalography (EEG) or magnetoencephalography signals [9-16] observable over a large network of brain areas [17-20] but predominantly in the temporal cortex.

Acoustic coordinated reset (CR) therapy is a soundbased therapy supported by neuromodulation principals [2123]. Utilizing the tonotopic organization of the auditory system, acoustic CR therapy uses sequences of acoustic tonal stimuli, namely, four different frequencies centered around 

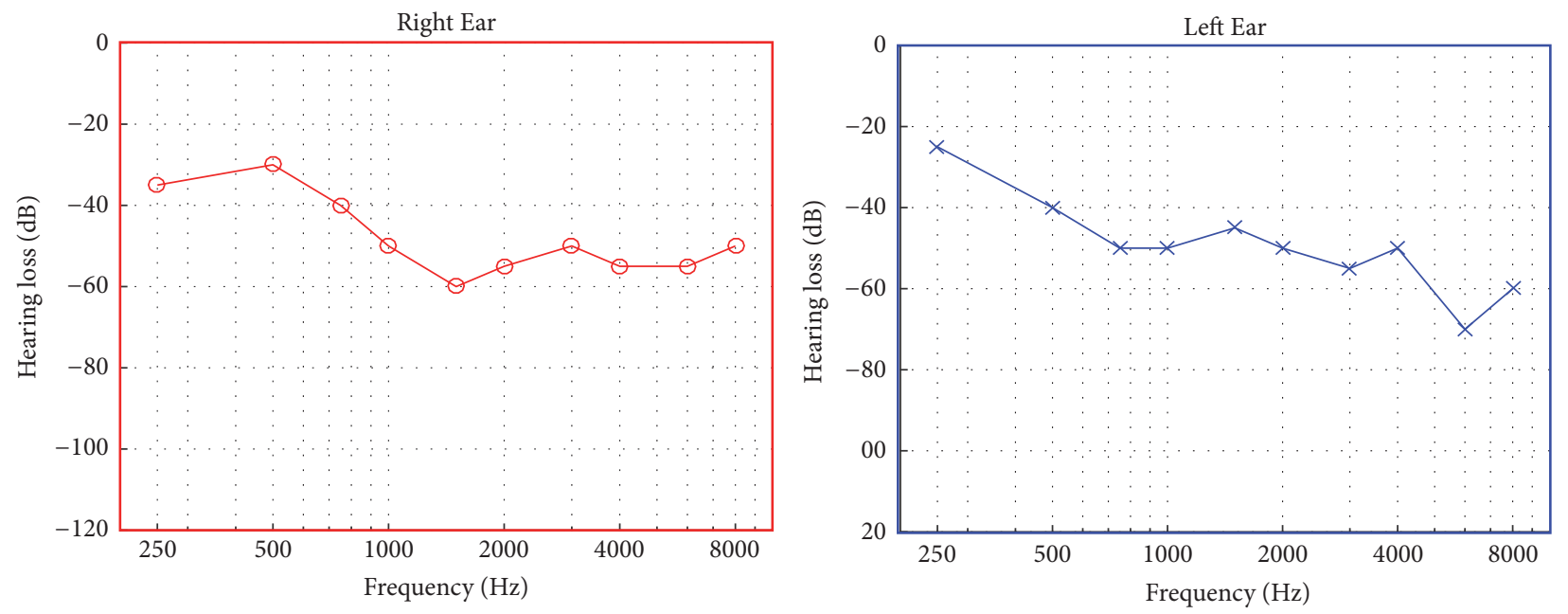

FIgURE 1: Audiograms of the patient obtained before starting the second treatment phase.

the characteristic frequency of the patient's tinnitus percept, delivered in nonsimultaneous sequences several hours per day for several weeks [14]. The stimuli target areas of pathological neural synchrony within the central auditory system and are intended to induce a sustained reduction of the strength of tinnitus-related synaptic connectivity among neurons within the affected neuronal population $[14,19]$ after therapy completion. This form of therapy has been tested in several clinical studies $[14,24,25]$. These studies have shown that statistically and clinically significant improvements in tinnitus symptoms were obtained in a variety of outcome measures including visual analog scales (VAS) for tinnitus loudness (reduction of 53\% during stimulation and $31 \%$ after stimulation), tinnitus annoyance (reduction of $49 \%$ during stimulation and $27 \%$ after stimulation), and tinnitus questionnaire results (reduction of $29 \%$ compared to baseline) $[14,15,26]$. The investigation of EEG activity has shown a significant decrease of abnormal EEG power spectra in the delta, theta, beta, and gamma bands to more normal levels $[14,16,27]$, a statistically significant decrease of effective connectivity within frequency bands [19], and a statistically significant decrease of cross-frequency coupling [28]. In a large observational study of 200 patients at 23 clinical sites a statistically and clinically significant improvement in Tinnitus Impairment Questionnaire scores (a reduction by up to $38 \%$ ) and in Clinical Global Improvement-Impression scales was observed (a reduction by up to 33\%) [24].

In these studies, the daily therapy signals were delivered by a custom device (T30 CR neurostimulator) connected to earphones by cables, an arrangement that is not ideal. The use of the therapy devices requires that hearing aid patients remove their hearing aids during therapy so the benefits of the hearing aids are removed during the therapy, an important consideration because a large percentage of patients with chronic primary tinnitus also have a degree of hearing loss that warrants hearing aids and the use of hearing aids in tinnitus patients itself can reduce tinnitus symptoms [29-31]. Furthermore, few studies have shown that sound therapies delivered by hearing aids were successful in alleviating effects of tinnitus [32,33].

We report results obtained by the delivery of acoustic CR therapy via hearing aids and results obtained by delivery via a separate proprietary sound system in a single hard to treat patient.

\section{Case Report}

One male patient (52 years) with a diagnosis of idiopathic chronic primary bilateral tinnitus participated in this case study. His tinnitus symptoms started in 2005 at the age of 41 years. He presented bilateral, sensorineural, high frequency hearing loss with a pure-tone average of $49 \mathrm{~dB} \mathrm{HL}$ in both ears (Figure 1).

2.1. Overview of Chronologic Sequence of Treatments. The patient wore hearing aids for 8 months prior to initial treatment with acoustic CR neuromodulation, relies on them for communication ability, and reported that they provide no benefits regarding tinnitus symptoms. The CR neuromodulation therapy was implemented for 19 weeks in 2012 with the standard acoustic stimuli delivered via a commercially available tinnitus device (T30 CR, ANM $\mathrm{GmbH}$, Cologne, Germany). The tinnitus therapy required removal of his hearing aids during the therapy. Subsequently (2015-2016), he was treated for 12 weeks with the same neuromodulation therapy device (T30 CR, Neurotherapies Reset GmbH, Jülich, Germany, a company of the Brook Henderson Group Limited) but connected to an external "streamer" (Phonak ComPilot,) that was a component of commercially available hearing aids (Phonak Audeo V50 Sonova AG, Stäfa, Switzerland), Figure 2.

The streamer had a sampling rate of $22 \mathrm{kHz}$ (stereo) and an audio bandwidth from $20 \mathrm{~Hz}$ to $10 \mathrm{kHz}$ (Datasheet Phonak ComPilot), while the hearing aid (Phonak Audeo V50) had a bandwidth from $100 \mathrm{~Hz}$ to $9.2 \mathrm{kHz}$. These bandwidth limitations, well known for conventional hearing aid signal 

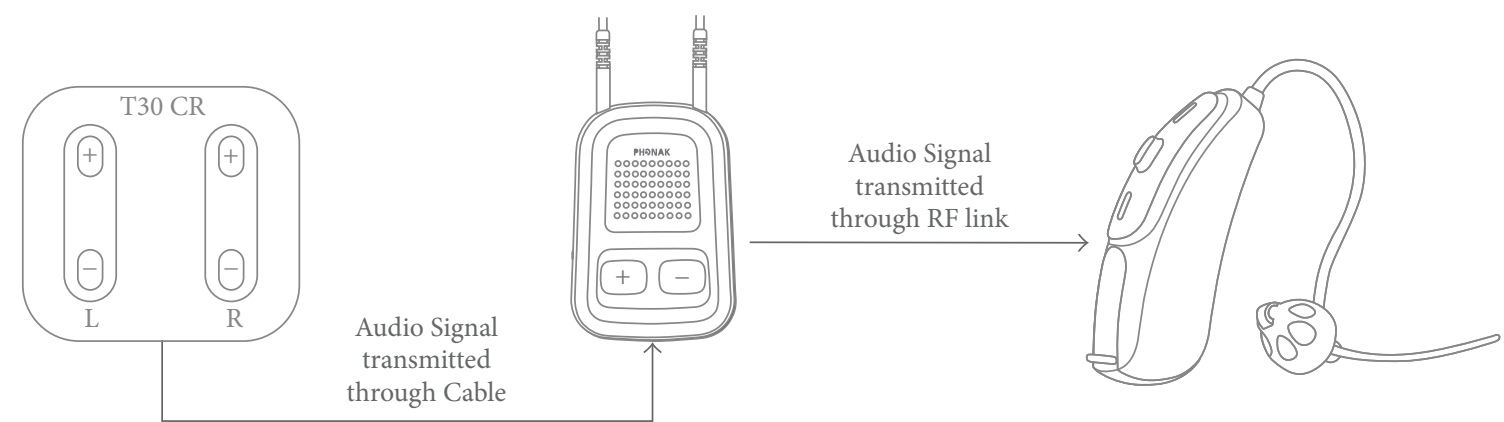

FIGURE 2: Device configuration. The tinnitus neuromodulation therapy device (neurostimulator T30 CR, left) applied the acoustic CR neuromodulation signals via cable to the "streamer" (Phonak ComPilot, middle) that in turn sent the therapy signals via radio frequency link to the two hearing aids (Phonak Audeo V50, right).

processing [34], may have additional limitations for the transfer of the CR therapy signals, especially in the high frequency range.

No further otolaryngology or audiology related issues were diagnosed while undergoing the two therapy phases and no pretreatments for tinnitus were reported except for the hearing aids that were fitted in July 2015. All treatments and hearing aid fittings were conducted by one of the authors (Mark Williams, Chief Audiologist at The Tinnitus Clinic, London, UK).

\subsection{Acoustic CR Neuromodulation and Outcome Measures.}

The standard acoustic CR neuromodulation therapy signals were used in both therapy periods and consisted of four pure tones determined by the pitch matched tinnitus frequency. The frequencies of the four therapy tones, two below and two above the tinnitus frequency, were a percentage of the tinnitus frequency, namely, from $77 \%$ to $140 \%$, presented sequentially in a randomized fashion at approximately $5 \mathrm{~dB}$ above the individual threshold at that frequency. The daily stimulation duration was 4-6 hours [14].

Tinnitus outcome measures used were the Tinnitus Handicap Inventory (THI) scores and the visual analog scale scores for tinnitus loudness $\left(\mathrm{VAS}_{\mathrm{L}}\right.$ on a scale from 0 to 100) and for tinnitus annoyance $\left(\operatorname{VAS}_{\mathrm{A}}\right.$ on a scale from 0 to 100) at baseline and after treatment. Please note that the sensitivity of the THI score has been challenged by Tyler et al. [35].

\subsection{First Phase of Treatment with Acoustic CR Neuromodula-} tion. In the first treatment phase (June 2012-November 2012) the measured tinnitus pitch fluctuated substantially with a mean pitch of $8040 \mathrm{~Hz}$ and standard deviation of $1400 \mathrm{~Hz}$. During this treatment phase, tinnitus pitch matching was performed using a bracketing method, similar to the wellknown Vernon-method [36]. Namely, at the six consecutive visits $7035 \mathrm{~Hz}, 9200 \mathrm{~Hz}, 6500 \mathrm{~Hz}, 9000 \mathrm{~Hz}, 6500 \mathrm{~Hz}$, and $10000 \mathrm{~Hz}$ were used for the programming of the acoustic CR neuromodulation pattern. During the first phase of treatment the patient reported that his tinnitus perception had started to become more atonal in nature and reported that he was gradually becoming less confident regarding the accuracy of his pitch matching ability (i.e., therefore he exhibited a lack of confidence about the reliability of the result and a marked decrease in salience of the tonal aspect). The baseline initial THI score was 28 points, the $\mathrm{VAS}_{\mathrm{L}}$ score was 80 points, and the $\mathrm{VAS}_{\mathrm{A}}$ score was 70 points. During each of five follow-up visits the therapy signal was readjusted with respect to the measured tinnitus frequency. After five months of therapy the THI (44 points), $\mathrm{VAS}_{\mathrm{L}}$ ( 90 points), and $\mathrm{VAS}_{\mathrm{A}}(80$ points) scores worsened by $57 \%, 13 \%$, and $14 \%$, respectively, Figure 3(a).

Another follow-up visit in February 2013 showed no change in tinnitus symptoms and therapy was stopped. In July 2015 hearing aids were fitted (Oticon Zest BTE digital instruments, Oticon A/S, Smørum, Denmark). The hearing aid was set up using a recent audiogram applying a national acoustic laboratory nonlinear prescription formula (NAL NL1) [37]. The patient had used hearing aids for the correction of his symmetrical presbycusis. No change in tinnitus symptoms was reported after wearing the hearing aids with no acoustic CR neuromodulation for 5 months (July 2015 to November 2015).

2.4. Second Phase of Treatment with Acoustic CR Neuromodulation. For the second treatment phase, the T30 CR device was connected via direct cable to a hearing aid streamer (Phonak ComPilot, Sonova AG, Stäfa, Switzerland) that wirelessly transmitted the therapy signals to hearing aids (Phonak Audeo V50, Sonova AG, Stäfa, Switzerland). The streamer sends an external audio signal (e.g., from an mp3 player, telephone, TV, or stereo system) through an external sound input to hearing aids that provide higher amplification customized to the patients hearing loss. The hearing aids (Phonak Audeo V50) were fitted with the same NAL NL1 prescription formula as used for the initial hearing aid (Oticon hearing aid Zest BTE digital instruments, Oticon A/S, Smørum, Denmark). This was done in order to ensure comparative amplification with the patient's original Oticon hearing aids used during phase one of treatment. Because the hearing loss was greater for high frequencies, in particular for frequencies above $6 \mathrm{kHz}$, the patient was not able to hear the highest stimulation tone, resulting in use of only the first three CR tones. Normally, this could cause the exclusion of the patient from treatment but was 


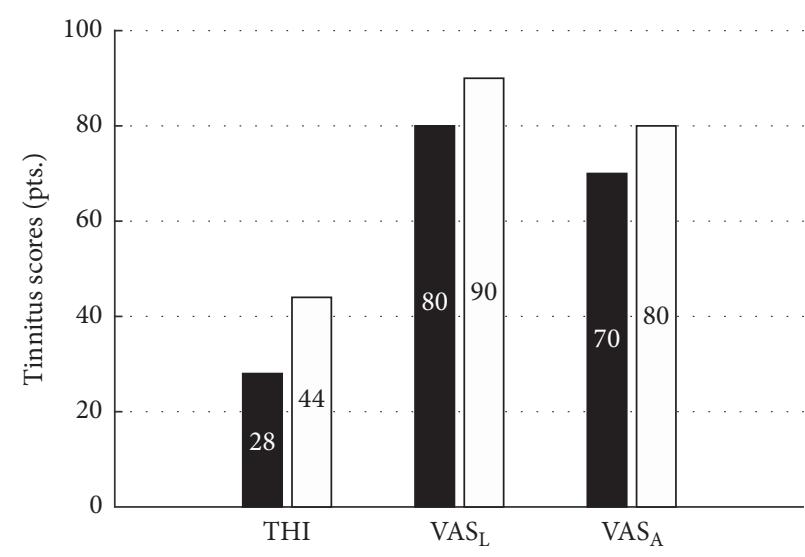

(a)

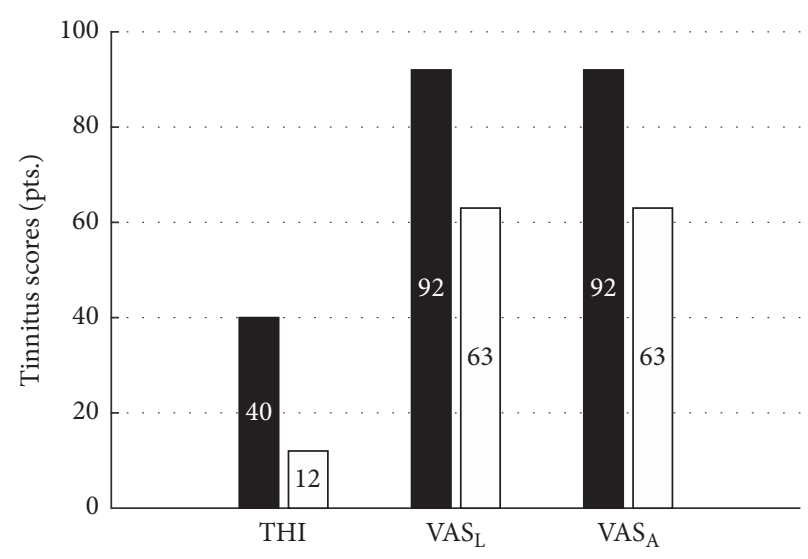

(b)

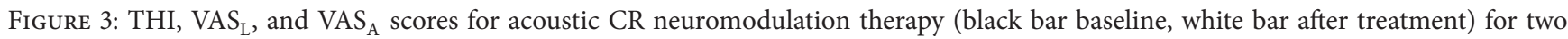
treatment phases. The first treatment phase (a) used only a CR neuromodulation device (T30 CR device with custom earphones only). The second treatment phase (b) used the same neuromodulation device (T30 CR) but combined with a streamer (Phonak ComPilot) wirelessly connected to hearing aids (Phonak Audeo V50).

accepted for this case report. The tinnitus pitch was quite stable during the second treatment phase with a lower mean pitch of $6260 \mathrm{~Hz}$ and a much smaller standard deviation of $150 \mathrm{~Hz}$. During this treatment phase, a newly developed automated and patented pitch matching method was utilized that integrated bracketing, similarity grading, a two-alternative forced-choice (2AFC) method, and fine-tuning into a single protocol of four distinct stages shown to improve pitch matching accuracy [38]. Namely, at the three consecutive visits $6109 \mathrm{~Hz}, 6109 \mathrm{~Hz}$, and $6421 \mathrm{~Hz}$ were used for the programming of the acoustic CR neuromodulation pattern. The baseline score for THI was 40 points, for $\mathrm{VAS}_{\mathrm{L}}$ was 92 points, and for $\mathrm{VAS}_{\mathrm{A}}$ was 92 points. During two follow-up visits the therapy signal was readjusted to the slightly varying tinnitus frequency. After three months of therapy a large improvement of tinnitus symptoms was observed; namely, the THI score was reduced by $70 \%$ to 12 points, the $\mathrm{VAS}_{\mathrm{L}}$ score was reduced by $32 \%$ to 63 points, and the $\mathrm{VAS}_{\mathrm{A}}$ score was reduced by $32 \%$ to 63 points, Figure $3(\mathrm{~b})$. The improvements of tinnitus symptoms were further substantiated by the additional results from another tinnitus questionnaire (TQ). The baseline TQ of 52 points was reduced by $35 \%$ to 34 points after 3 months of acoustic CR therapy applied through a hearing aid.

\section{Discussion}

During application of acoustic CR neuromodulation via a hearing aid a significant reduction in tinnitus symptoms was observed as documented by substantial decreases in $\mathrm{VAS}_{\mathrm{L}}$, $\mathrm{VAS}_{\mathrm{A}}$, and TQ scores. The magnitude of the hearing loss and the variable pitch matching results may have contributed to the unsuccessful initial acoustic CR neuromodulation treatment attempt. The application of acoustic CR neuromodulation through the hearing aids was well accepted and no side effects were observed throughout the intervention.
At the time of writing the majority of hearing instrument manufacturers provide aids with sound therapy features that can be utilized for a number of different therapeutic purposes and may serve to reduce the distress associated with tinnitus perception. Examples of these features include sound signals that are intended to be conducive to relaxation [39]; alternatively, others can be applied in order to provide some form of sound enrichment or masking. A low number of smallscale studies have reported that these systems can be useful for the management of tinnitus, with the supplementation of appropriate counseling [33].

Results obtained during this case report are encouraging from several clinical and technical standpoints. First, this approach is also applicable for tinnitus patients with sufficient hearing loss to warrant the use of hearing aids. Second, this approach allows simultaneously tinnitus therapy and hearing aid benefits. Third, this approach allows improved acoustic CR neuromodulation therapy signal control because of the inherent further amplification offered by the hearing aids used to treat the existing hearing loss. Fourth, this approach likely will result in higher compliance with the therapy regime because the primary device already is being worn. Fifth, the continued use of the hearing aid beyond acoustic CR neuromodulation therapy might help to stabilize the obtained therapeutic effects. Finally, this approach will allow easy implementation of additional CR therapy should the tinnitus return or worsen in the future.

There are some limitations with this approach. First, current hearing aids have a restricted frequency range that will limit the delivery of high frequency stimulation tones with high enough levels in cases of high frequency tinnitus. Second, the available data did not fully explain why the patient did not respond to CR therapy without hearing aid support in the first phase of the experiment. This might have occurred because of inadequate and imprecise pitch matching (see Section 2.3) or because of the substantial hearing loss 
close to the used stimulation tones. The slight worsening of the tinnitus symptoms might be linked to the patient's emotional reaction to not experiencing obvious improvement during therapy. Third, there may be some inconvenience related to the need for an optional "streamer" module. As an alternative, to cope with the latter disadvantage for further patients within this continued case series, devices are being developed [38] that allow the direct streaming of therapy signals to hearing aids wirelessly as is done for other sound therapies involving noise generators $[32,33]$.

\section{Conclusion}

In a single case, we were able to show that acoustic CR neuromodulation can be implemented effectively through contemporary hearing aids to allow the simultaneous benefits of hearing aid use and therapy delivery. This approach appeared to not negatively influence normal hearing aid functionality, increase the environments in which therapy can be delivered, and potentially increase likelihood of compliance with the tinnitus therapy regimen. Since only one case has been investigated so far, the results are clearly not yet conclusive. This case report encourages a more comprehensive investigation of the integration of acoustic CR therapy delivery with hearing aids including understanding the effects of nonlinear amplification, harmonic intermodulation, and temporal distortions inherent in contemporary hearing aids.

\section{Conflicts of Interest}

Christian Hauptmann is employed by DESYNCRA Operating $\mathrm{GmbH}$, Bad Neuenahr-Ahrweiler (part of DESYNCRA Technologies Ltd, London, UK). He worked with Juelich Research Center between 2002 and 2016 and has received research funding from the European Community, the Federal Ministry of Education and Research (Germany), the Deutsche Forschungsgemeinschaft, and the Helmholtz Association. Mark Williams is an audiologist with a contractual relationship with The Tinnitus Clinic Ltd., the UK distributer for the Acoustic Coordinated Reset Neuromodulation therapy device. He also is a consultant for Brook Henderson Group (DESYNCRA Technologies Limited). Federica Vinciati is a neuroscientist and had a contractual relationship with DESYNCRA Technologies Ltd, London, UK from January 2016 to February 2017. Markus Haller is employed by DESYNCRA Technologies Ltd, London, UK. The product under investigation is marketed by Neurotherapies Reset $\mathrm{GmbH}$, Jülich, Germany, a company of the Brook Henderson Group Limited. A new platform for the application of acoustic CR neuromodulation utilizing a mobile device (Hauptmann et al. 2016) has been developed by Juelich Research Center and DESYNCRA Tinnitus Limited (a company of the Brook Henderson Group Limited).

\section{Authors' Contributions}

Christian Hauptmann and Mark Williams equally contributed to this case report.

\section{Acknowledgments}

The study was supported by DESYNCRA Technologies Ltd. The authors thank the Tinnitus Clinic (TTC) for providing access to their clinical premises and their support for this study. The authors thank Peter Tass and Gerald Popelka for fruitful input and discussions during manuscript preparation.

\section{References}

[1] J. J. Eggermont and L. E. Roberts, "The neuroscience of tinnitus," Trends in Neurosciences, vol. 27, no. 11, pp. 676-682, 2004.

[2] A. Axelsson and A. Ringdahl, "Tinnitus-a study of its prevalence and characteristics," British Journal of Audiology, vol. 23, no. 1, pp. 53-62, 1989.

[3] B. Langguth, "Tinnitus: the end of therapeutic nihilism," The Lancet, vol. 379, no. 9830, pp. 1926-1928, 2012.

[4] D. E. Tunkel, C. A. Bauer, G. H. Sun et al., "Clinical practice guideline: Tinnitus," Otolaryngology-Head and Neck Surgery, vol. 151, no. 2, pp. S1-S40, 2014.

[5] P. Martinez Devesa, A. Waddell, R. Perera, and M. Theodoulou, "Cognitive behavioural therapy for tinnitus," Cochrane Database of Systematic Reviews, no. 1, Article ID CD005233, 2007.

[6] D. J. Hoare, M. Edmondson-Jones, M. Sereda, M. A. Akeroyd, and D. Hall, "Amplification with hearing aids for patients with tinnitus and co-existing hearing loss," The Cochrane Database of Systematic Reviews, vol. 1, Article ID CD010151, 2014.

[7] J. Hobson, E. Chisholm, and A. El Refaie, "Sound therapy (masking) in the management of tinnitus in adults," Cochrane Database of Systematic Reviews, vol. 11, Article ID CD006371, 2012.

[8] R. R. Llinás, U. Ribary, D. Jeanmonod, E. Kronberg, and P. P. Mitra, "Thalamocortical dysrhythmia: a neurological and neuropsychiatric syndrome characterized by magnetoencephalography," Proceedings of the National Academy of Sciences of the United States of America, vol. 96, no. 26, pp. 15222-15227, 1999.

[9] N. Weisz, S. Moratti, M. Meinzer, K. Dohrmann, and T. Elbert, "Tinnitus perception and distress is related to abnormal spontaneous brain activity as measured by magnetoencephalography," PLoS Medicine, vol. 2, no. 6, article e153, 2005.

[10] J. J. Eggermont and P. A. Tass, "Maladaptive neural synchrony in tinnitus: origin and restoration," Frontiers in Neurology, vol. 6, article 29, 2015.

[11] K. Dohrmann, T. Elbert, W. Schlee, and N. Weisz, "Tuning the tinnitus percept by modification of synchronous brain activity," Restorative Neurology and Neuroscience, vol. 25, no. 3-4, pp. 371378, 2007.

[12] N. Kahlbrock and N. Weisz, "Transient reduction of tinnitus intensity is marked by concomitant reductions of delta band power," BMC Biology, vol. 6, article 4, 2008.

[13] M. Ortmann, N. Müller, W. Schlee, and N. Weisz, "Rapid increases of gamma power in the auditory cortex following noise trauma in humans," European Journal of Neuroscience, vol. 33, no. 3, pp. 568-575, 2011.

[14] P. A. Tass, I. Adamchic, H. J. Freund, T. von Stackeberg, and C. Hauptmann, "Counteracting tinnitus by acoustic coordinated reset neuromodulation," Restorative Neurology and Neuroscience, vol. 31, no. 3, pp. 235-237, 2013.

[15] I. Adamchic, B. Langguth, C. Hauptmann, and P. A. Tass, "Psychometric evaluation of visual analog scale for the assessment 
of chronic tinnitus," American Journal of Audiology, vol. 21, no. 2, pp. 215-225, 2012.

[16] I. Adamchic, T. Toth, C. Hauptmann, and P. A. Tass, "Reversing pathologically increased EEG power by acoustic coordinated reset neuromodulation," Human Brain Mapping, vol. 35, no. 5, pp. 2099-2118, 2014.

[17] W. Schlee, N. Weisz, O. Bertrand, T. Hartmann, and T. Elbert, "Using auditory steady state responses to outline the functional connectivity in the tinnitus brain," PLoS ONE, vol. 3, no. 11, Article ID e3720, 2008.

[18] D. De Ridder, A. B. Elgoyhen, R. Romo, and B. Langguth, "Phantom percepts: tinnitus and pain as persisting aversive memory networks," Proceedings of the National Academy of Sciences of the United States of America, vol. 108, no. 20, pp. 8075-8080, 2011.

[19] A. N. Silchenko, I. Adamchic, C. Hauptmann, and P. A. Tass, "Impact of acoustic coordinated reset neuromodulation on effective connectivity in a neural network of phantom sound," NeuroImage, vol. 77, pp. 133-147, 2013.

[20] W. Sedley, P. E. Gander, S. Kumar et al., "Intracranial mapping of a cortical tinnitus system using residual inhibition," Current Biology, vol. 25, no. 9, pp. 1208-1214, 2015.

[21] P. A. Tass, "A model of desynchronizing deep brain stimulation with a demand-controlled coordinated reset of neural subpopulations," Biological Cybernetics, vol. 89, no. 2, pp. 81-88, 2003.

[22] P. A. Tass and M. Majtanik, "Long-term anti-kindling effects of desynchronizing brain stimulation: a theoretical study," Biological Cybernetics, vol. 94, no. 1, pp. 58-66, 2006.

[23] P. A. Tass and O. V. Popovych, "Unlearning tinnitus-related cerebral synchrony with acoustic coordinated reset stimulation: theoretical concept and modelling," Biological Cybernetics, vol. 106, no. 1, pp. 27-36, 2012.

[24] C. Hauptmann, A. Ströbel, M. Williams et al., "Acoustic coordinated reset neuromodulation in a real life patient population with chronic tonal tinnitus," BioMed Research International, vol. 2015, Article ID 569052, 2015.

[25] M. Williams, C. Hauptmann, and N. Patel, "Acoustic CR neuromodulation therapy for subjective tonal tinnitus: a review of clinical outcomes in an independent audiology practice setting," Frontiers in Neurology, vol. 6, article 54, 2015.

[26] I. Adamchic, P. A. Tass, B. Langguth et al., "Linking the Tinnitus Questionnaire and the subjective Clinical Global Impression: which differences are clinically important?" Health and Quality of Life Outcomes, vol. 10, article 79, 2012.

[27] I. Adamchic, C. Hauptmann, and P. A. Tass, "Changes of oscillatory activity in pitch processing network and related tinnitus relief induced by acoustic CR neuromodulation," Frontiers in Systems Neuroscience, vol. 6, article 18, 2012.

[28] I. Adamchic, B. Langguth, C. Hauptmann, and P. A. Tass, "Abnormal cross-frequency coupling in the tinnitus network," Frontiers in Neuroscience, vol. 8, article 284, 2014.

[29] G. S. Shekhawat, G. D. Searchfield, and C. M. Stinear, "Role of hearing aids in tinnitus intervention: a scoping review," Journal of the American Academy of Audiology, vol. 24, no. 8, pp. 747762, 2013.

[30] S. Kochkin and R. Tyler, "Tinnitus treatment and the effectiveness of hearing aids: hearing care professional perceptions," The Hearing Review, vol. 15, no. 13, pp. 14-18, 2008.

[31] H. Jalilvand, A. Pourbakht, and H. Haghani, "Hearing aid or tinnitus masker: which one is the best treatment for blastinduced tinnitus? The results of a long-term study on 974 patients," Audiology and Neurotology, vol. 20, no. 3, pp. 195-201, 2015.

[32] G. M. Dos Santos, R. F. Bento, I. R. T. De Medeiros, J. Oiticcica, E. C. Da Silva, and S. Penteado, "The influence of sound generator associated with conventional amplification for tinnitus control: randomized blind clinical trial," Trends in Hearing, vol. 18, 2014.

[33] J. A. Henry, M. Frederick, S. Sell, S. Griest, and H. Abrams, "Validation of a novel combination hearing aid and tinnitus therapy device," Ear and Hearing, vol. 36, no. 1, pp. 42-52, 2015.

[34] G. R. Popelka, B. C. J. Moore, R. R. Fay, and A. N. Popper, Eds., Hearing Aids, Springer International Publishing, 2016.

[35] R. S. Tyler, J. Oleson, W. Noble, C. Coelho, and H. Ji, “Clinical trials for tinnitus: study populations, designs, measurement variables, and data analysis," Progress in Brain Research, vol. 166, pp. 499-509, 2007.

[36] J. A. Vernon and M. B. Meikle, "Measurement of tinnitus: an update," in Tinnitus: Pathophysiology, Diagnosis and Treatment, M. Kitahara, Ed., pp. 36-52, Igaku-Shoin, Tokyo, Japan, 1988.

[37] D. Byrne, H. Dillon, T. Ching, R. Katsch, and G. Keidser, "NALNL1 procedure for fitting nonlinear hearing aids: characteristics and comparisons with other procedures," Journal of the American Academy of Audiology, vol. 12, no. 1, pp. 37-51, 2001.

[38] C. Hauptmann, A. Wegener, H. Poppe, M. Williams, G. Popelka, and P. A. Tass, "Validation of a mobile device for acoustic coordinated reset neuromodulation tinnitus therapy," Journal of the American Academy of Audiology, vol. 27, no. 9, pp. 720-731, 2016.

[39] R. Sweetow, "The use of fractal tones in tinnitus patient management," Noise and Health, vol. 15, no. 63, pp. 96-100, 2013. 


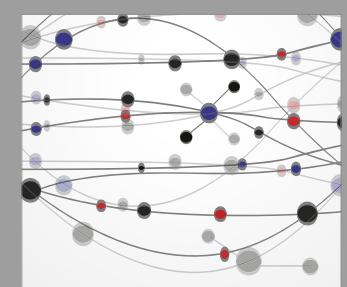

The Scientific World Journal
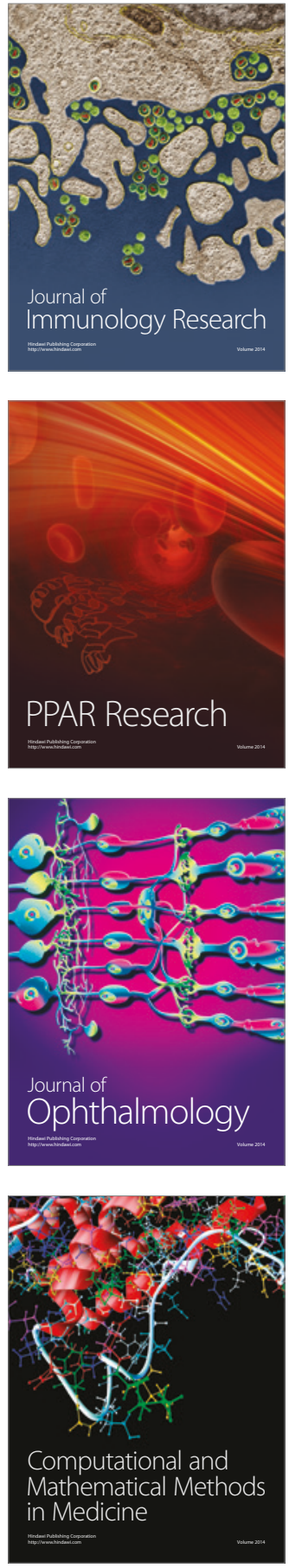

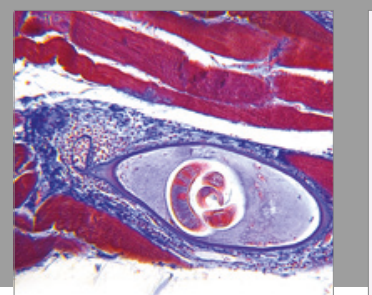

Gastroenterology Research and Practice
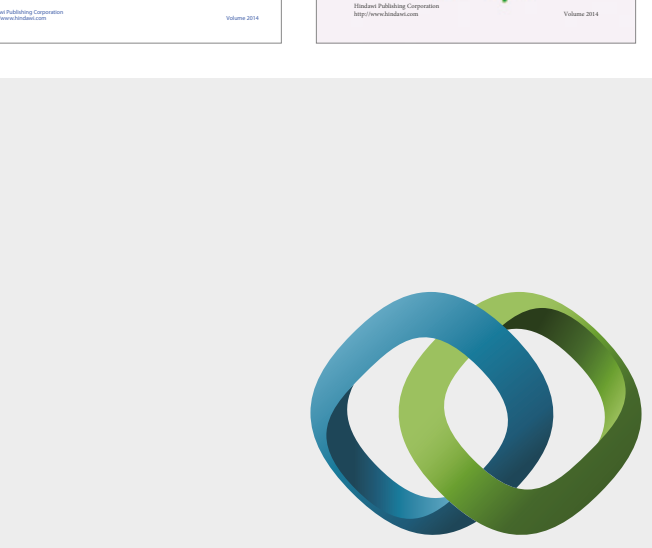

\section{Hindawi}

Submit your manuscripts at

https://www.hindawi.com
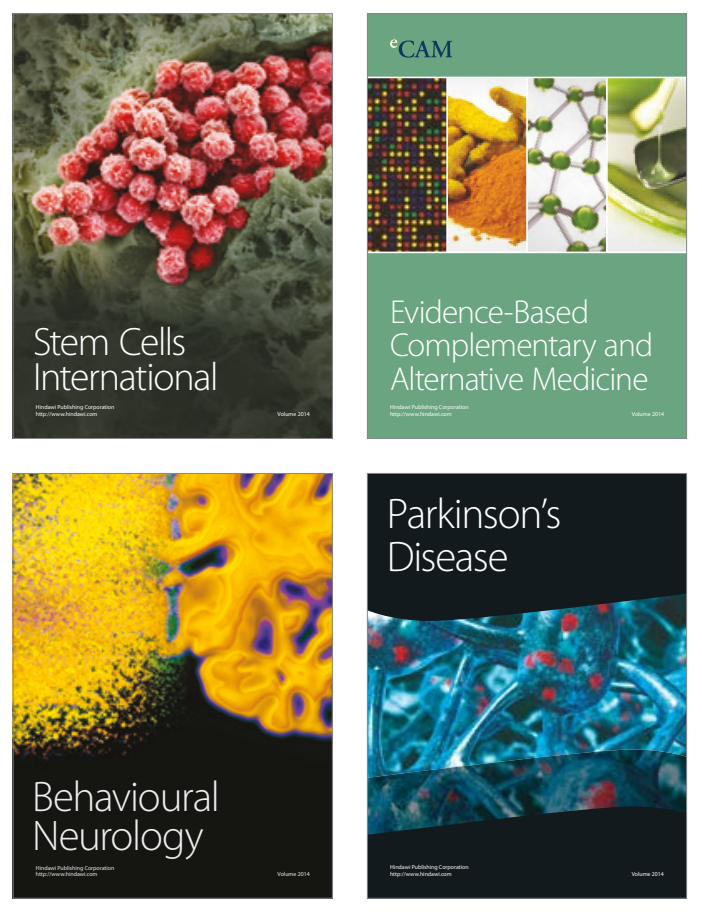
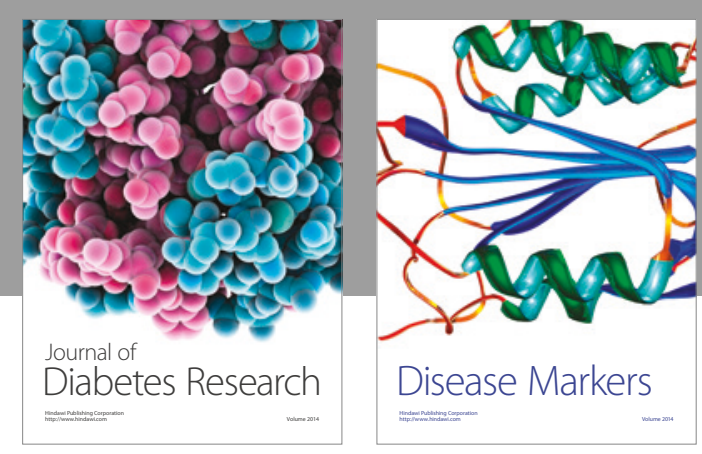

Disease Markers
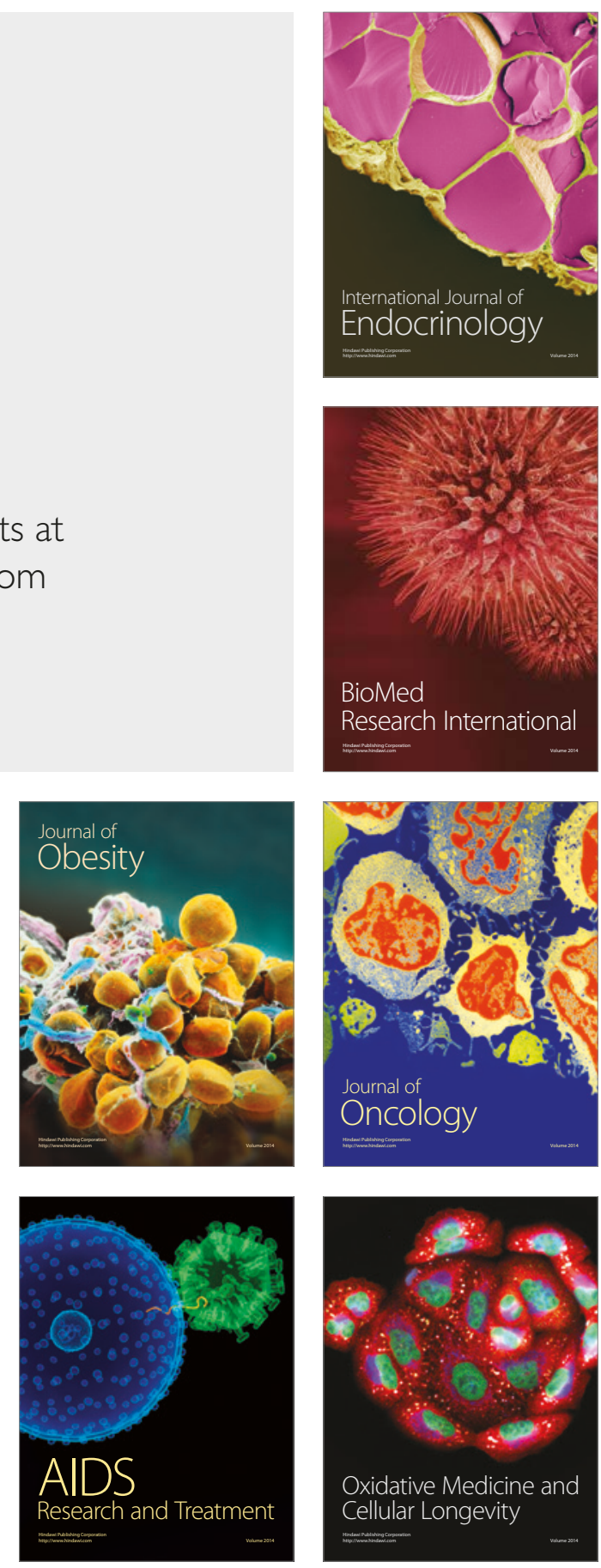